\title{
TMAO reductase, a biomarker for gut permeability defect induced inflammation, in mouse model of chronic kidney disease and dextran sulfate solution-induced mucositis
}

\author{
Sompong Boonhai, ${ }^{1}$ Kanthika Bootdee, ${ }^{2}$ Wilasinee Saisorn, ${ }^{2}$ Kullaya Takkavatakarn, ${ }^{3}$ Patita Sitticharoenchai, ${ }^{4}$ \\ Somkanya Tungsanga, ${ }^{3}$ Khajohn tiranathanagul, ${ }^{3}$ Asada Leelahavanichkul ${ }^{2,3,5}$
}

\begin{abstract}
Background: The excretion of trimethylamine N-oxide (TMAO) (uremic toxin) into the intestine might be enhanced, due to the limited renal elimination in chronic kidney disease (CKD), possibly induced TMAO reductase (a TMAO-neutralizing enzyme) in gut bacteria. Detection of TMAO reductase in serum could be used as a biomarker of gut permeability defect.
\end{abstract}

Objective: To explore the correlation between serum TMAO reductase, gut leakage, and systemic inflammation in CKD.

Methods: Mouse models of gut leakage; including 5/6 nephrectomy-induced chronic kidney disease (CKD), a model without colitis, and $1.5 \%$ dextran sulfate solution (DSS), a colitis model, were performed. In parallel, serum samples from patients with chronic hemodialysis $(n=48)$ and the healthy control $(n=20)$ were analyzed.

Results: Gut-leakage (FITC-dextran, endotoxemia, and reduced intestinal tight junction protein) was detected in both CKD and DSS models. While TMAO reductase and TMAO were elevated in the serum of both mouse models and patients, TMAO reductase correlated with TMAO, gut- leakage, and serum IL-6 only in mice but not in patients. Notably, endotoxemia was used as a surrogate marker of gut leakage in patients. In patients, TMAO reductase and TMAO did not correlate with serum IL-6 and vascular complications using the ankle-brachial index and cardio-ankle vascular index.

Conclusions: Serum TMAO reductase was elevated in CKD mice and patients with CKD. Serum TMAO reductase was correlated with TMAO and gut-leakage only in mice but not in patients. Further studies in patients are needed to determine the benefit of serum TMAO reductase in patients with CKD.

Key words: TMAO reductase, biomarker, inflammation, kidney disease, mucositis

\section{From:}

${ }^{1}$ Master of Science Program in Molecular Science of Medical Microbiology and Immunology, Department of Transfusion Medicine and Clinical Microbiology Faculty of Allied Health Sciences, Chulalongkorn University

Department of Microbiology, King Chulalongkorn Memorial Hospital, Thai Red Cross Society, Bangkok, Thailand

3 Division of Nephrology, Department of Medicine, King Chulalongkorn Memorial Hospital, Thai Red Cross Society and Faculty of Medicine, Chulalongkorn University

${ }^{4}$ Division of Cardiology, Department of Medicine,

Faculty of Medicine, King Chulalongkorn Memorial Hospital, Bangkok, Thailand

Translational Research in Inflammation and Immunology Research Unit (TRIRU), Department of Microbiology,

Chulalongkorn University, Bangkok, Thailand
Corresponding author:

Asada Leelahavanichkul

Immunology Unit, Department of Microbiology,

Chulalongkorn University, Bangkok 10330, Thailand

E-mail: aleelahavanit@gmail.com

\section{Introduction}

A single layer of epithelial cells, held together by epithelial tight junctions (TJ), is the major intrinsic mucosal defense barrier between the host and gut organisms. Indeed, normal TJ only allows the passive movement of molecules that smaller than $0.6 \mathrm{kDa}$ through paracellular transport. ${ }^{1}$ Gut mucosa injury induces gut permeability defect allows the translocation of organismal molecules from the gut into blood circulation 
refer to as "gut leakage". ${ }^{2-6}$ Because of the foreignness to host of microbial molecules, most of these molecules from gut translocation induce systemic inflammatory responses. ${ }^{5}$ Due to the dominant abundance of Gram-negative bacteria in the gut, gut translocation of lipopolysaccharide (LPS), referred to as endotoxemia, demonstrates several adverse effects to the host in both infectious diseases (bacterial endotoxemia), or non-infection diseases (metabolic endotoxemia). ${ }^{7}$ Endotoxemia without active infection is used as a biomarker for gut leakage because LPS is too large to pass through TJ (MW 50$100 \mathrm{kDa}) .^{2}$ The detection of gut leakage using endotoxemia is easier than the standard method with detection of the non-absorbable carbohydrates in urine or serum after oral ingestion. ${ }^{8}$ However, several non-gut-derived factors could be responsible for endotoxemia including occult infections and contaminated intravenous fluid. Hence, other bacterial products might be interesting to use as gut leakage biomarkers.

Chronic kidney disease (CKD) is a major health care problem worldwide ${ }^{9}$ with a possibility of endotoxemia from gut translocation. ${ }^{10}$ Accordingly, the uremic toxin is one of the major causes of intestinal TJ damage ${ }^{11}$ that is responsible for uremia-induced gut leakage. Interestingly, trimethylamine $\mathrm{N}$-oxide (TMAO), the amine oxides formed in the liver, is a metabolite of trimethylamine (TMA) which is produced from dietary phosphatidylcholine by bacteria in the gut. ${ }^{12}$ As such, TMAO, is small enough for the passive transportation through TJ into blood circulation ${ }^{12}$ that worsens renal function. ${ }^{13}$

While bacteria in the gut produce TMAO, TMAO reductase $(\mathrm{MW}$ at $172 \mathrm{kDa}$ ) is a bacterial enzyme that metabolizes $\mathrm{TMAO}^{14,15}$ which normally does not pass through the healthy intestinal barrier. ${ }^{1}$ Then the detection of TMAO reductase in blood might be associated with gut permeability defect. Different from the limitation of endotoxemia as a biomarker for gut leakage (including the LPS contamination during hemodialysis), the detection of serum TMAO reductase might be more specific to gut permeability defect. Moreover, it is wellknown that TMAO is associated with arterial complications in patients with CKD. ${ }^{16-18}$ If TMAO reductase is associated with TMAO level, TMAO reductase might also be a biomarker for CKD vascular complications. Due to the easier method for the detection of TMAO reductase (using ELISA) compared with TMAO (by mass-spectrometry), the utilization of TMAO reductase might be more affordable in regular clinical practice. Hence, we performed mouse models to explore the utilization of TMAO reductase in mouse models of CKD (5/6 nephrectomy) and dextran sulfate solution (DSS) induced mucositis and in serum from patients with CKD.

\section{Materials and Methods \\ Animal and animal models}

The animal study protocol following the National Institutes of Health (NIH), USA was approved by the Institutional Animal Care and Use Committee from the Faculty of Medicine, Chulalongkorn University, Bangkok, Thailand (SST 022/ 2561). C57BL/6 mice were purchased from Nomura Siam International (Pathumwan, Bangkok, Thailand). For the induction of chronic kidney disease (CKD), 5/6 nephrectomy $(\mathrm{Nx})$ was performed in 8 -wk-old male mice with two stages of operation under isoflurane anesthesia following the previous publications. ${ }^{19,20}$ Briefly, the resection of the upper and lower pole of the left kidney was performed through the left flank incision with the microfibrillar collagen hemostasis (Avitene, Davol, Cranston, RI). At 1 week later, the entire right kidney was removed via a right flank incision. For the sham-operated mice, the flank incisions were performed without renal resection. In parallel, a model of the direct mucosal injury, DSS-induced mucositis, was conducted as previously. ${ }^{6,21,22}$ In brief, DSS (Sigma-Aldrich, St. Louis, MO, USA) was diluted into the drinking water of 8 -wk-old male mice at the concentration of $1.5 \%(\mathrm{v} / \mathrm{v})$, and the regular drinking water was used for the control group. Then, CKD (and sham) mice and DSS (and water control) mice were sacrificed at 3 months after right nephrectomy and 7 days post-DSS, respectively. Mice were sacrificed with cardiac puncture under isoflurane anesthesia with the collection of blood and ascending colons (distal to the caecum). Serum was kept at $-80^{\circ} \mathrm{C}$ until used and ascending colon samples were prepared in 10\% formalin for histology and in Cryogel (Leica Biosystems, Richmond, IL, USA) for the immunofluorescent staining of the tight junction protein.

\section{Analysis of mouse samples and gut leakage determination}

Liver injury was determined by glutamic-oxaloacetic transaminase (SGOT) (EASTR-100) and glutamate-pyruvate transaminase (SGPT) (EALT-100) (BioAssay, Haywood, CA, USA). Renal injury was indicated by blood urea nitrogen (BUN) (QuantiChrom Urea Assay; DIUR-100) and serum creatinine (QuantiChrom Creatinine Assay; DICT-500) (BioAssay). Enzyme-linked immunosorbent assay (ELISA) for serum IL-6 (PeproTech, Rocky Hill, NJ, USA) and trimethylamine-N-oxide (TMAO) reductase (MBS7254609) (MyBioSource, San Diego, CA, USA) were used. On the other hand, gut leakage was determined by i) fluorescein isothiocyanate dextran (FITC-dextran) assay, ii) endotoxemia, iii) tight junction molecule detection using immunofluorescence, and iv) staining for enterocyte apoptosis. The detection of FITC-dextran, a nonabsorbable molecule, in serum with Fluorospectrometer (NanoDrop 3300; Thermo Scientific, Wilmington, DE, USA) ${ }^{23}$ at $3 \mathrm{~h}$ after an oral administration of $12.5 \mathrm{mg}$ FITC-dextran (4.4 kDa) (FD4; Sigma-Aldrich) indicates gut permeability defect. ${ }^{24}$ Serum endotoxin (LPS) was evaluated by HEK-Blue LPS Detection Kit 2 (InvivoGen, San Diego, CA, USA) ${ }^{25}$ with the limit of detection (LOD) at less than $0.01 \mathrm{EU} / \mathrm{mL}$.

Furthermore, the intestinal tight junctions of ascending colon were evaluated following the previous publications ${ }^{3,26}$ due to the dominant colon injury in DSS-induced mucositis. ${ }^{22}$ As such, samples in Cryogel (Leica Biosystems, Richmond, IL, USA) were prepared into $5 \mu \mathrm{m}$-thick acetone-fixed sections and were stained by primary antibody against enterocyte tight junction molecules Claudin-1 (catalog no. 71-7800; Thermo Fisher Scientific, Waltham, MA, USA) with secondary green fluorescent antibodies (Alexa Fluor 488) (Life Technologies, Carlsbad, CA, USA). Then, the slides were washed and stained with 4',6'-diamidino-2-phenylindole (DAPI; BioLegend, USA) $(1: 1,000)$ for $15 \mathrm{~min}$, mounted (Prolong; Life Technologies), and were visualized with a Zeiss LSM 800 confocal microscope (Carl Zeiss, USA). Additionally, enterocyte apoptosis 
was determined by immunohistochemistry as a possible cause of gut leakage. Accordingly, 10\% formalin-fixed paraffin-embedded sections of the colon were stained with anti-cleaved caspase-3 (Asp175) (catalog no. 9661; Cell Signaling Technology, Beverly, MA, USA) for $1 \mathrm{~h}$ before incubation with secondary biotinylated goat anti-rabbit antibody (Cell Signaling Technology) for $30 \mathrm{~min}$ before visualization. The semiquantitative analysis of apoptotic cells from 10 random fields per slide was evaluated, by 2 examiners in a blinded fashion, with the average number of enterocytes with apoptosis counted at a $200 \times$ magnification and expressed as the number of positive cells per high-power field.

\section{Serum trimethylamine-N-oxide (TMAO)}

Serum TMAO was determined by liquid chromatography-mass spectrometry (LC-MS/MS) following a previous publication $^{27}$ using a silica column (Luna silica; 00G-4274-E0, Phenomenex, Torrance, CA) at a flow rate of $0.8 \mathrm{~mL} / \mathrm{min}$ with a 4 LC-20AD Shimadazu pump system, SIL-HTC autosampler and dual column switching valve system (MXP7900, IDEX Health \& Science, Oak Harbor, WA ${ }^{28}$ interfaced with a Xevo ${ }^{\circ}$ TQ-S mass spectrometer (Waters Ges.m.b.H USA.). Electrospray ionization in positive-ion mode with multiple reaction monitoring (MRM) of precursor and characteristic product ion transitions of $\mathrm{m} / \mathrm{z} 76 \rightarrow 58 \mathrm{amu}$ and $85 \rightarrow 66 \mathrm{amu}$, respectively, were performed. The parameters for the ion monitoring were as follows: Spray voltage, $4.5 \mathrm{kV}$; Curtain gas, 15; GS1, 60; GS2, 50; CAD gas, medium; DP, 60; CE, 25.0 volts for TMAO and 28.3 volts for d9-TMAO (internal standard); CXP, 10; EP, 10. Nitrogen (99.95\% purity) was used. Various concentrations of non-isotopically labeled TMAO standards were spiked into control plasma to prepare the calibration curves. The internal standard d9-TMAO was used for quantification as well as to calculate the recovery rate of TMAO.

\section{Patient data}

Serum of patients without residual renal function in a regular chronic hemodiafiltration program (3 times a week) after 2 days of the dialysis free period from the Nephrology Unit of the King Chulalongkorn Memorial Hospital, Bangkok, Thailand or the healthy volunteers were collected following the approved protocol (NBC 18/2020) by the Ethical Institutional Review Board, Faculty of Medicine, Chulalongkorn University, according to the Declaration of Helsinki, with written informed consent from each patient. For the demographic data of the healthy control ( $\mathrm{n}=20 ; 40 \%$ male) and patients $(\mathrm{n}=$ 48 ; $46 \%$ male) in mean \pm standard deviation (SD) were; average age, $57 \pm 15$ vs. $65 \pm 12$ years old; height, $171 \pm 10$ vs. $160 \pm 9 \mathrm{~cm}$ and body mass index (BMI), $28 \pm 10$ vs $25 \pm 8$. The dialysis vintage of patients and dry weight at the time of the sample collection were $11 \pm 5$ years and $61 \pm 15 \mathrm{~kg}$. The underlying diseases of the patients were diabetic nephropathy $(50 \%)$, hypertension (17\%), Glomerular diseases (8\%), and unknown (25\%). The serum samples were collected after 2 days of dialysis free in all patients as the representatives of the chronic kidney disease group. The exclusion criteria were patients with current infection or history of infection within 2 months, pregnancy, liver cirrhosis, active lupus, or other auto-immune diseases. The analysis of patient samples was based on the hospital service using i) Alinity ci-series Integrated Clinical Chemistry and Immunoassay (Abbott, Abbott Park, Illinois, USA) for detection of BUN, Creatinine, SGOT, and SGPT, ii) Cobas 8000 c502 (Roche Diagnostics, Brentwood, TN, USA) for $\beta 2$-microglobulin $(\beta 2 \mathrm{M})$ and C-reactive protein (CRP). Serum TMAO and TMAO reductase were determined by LC-MS/MS and the ELISA (MyBioSource), respectively, as mentioned above. Human cytokines (IL-6 and TNF- $\alpha$ ) and LPS were measured by ELISA (88-7066 and 887346; Thermo Fisher Scientific) and HEK-Blue LPS Detection Kit 2 (InvivoGen). In parallel, ankle-brachial Index (ABI) and cardio-ankle vascular index (CAVI) were used to determine the vascular complications using a portable ultrasonography-based machine (VaSera VS-200; Fukuda-Denshi Company, Tokyo, Japan) as published articles..$^{29,30}$ Accordingly, ABI is calculated by the highest systolic pressure on the foot of that side/average of the highest pressure from both arms, while CAVI score was calculated by the machine. The average of $\mathrm{ABI}$ and CAVI from left and right-sided measurements were used to determine the correlation with serum indicators. The ABI score $<0.9$ and 0.91-0.99 are peripheral arterial disease and borderline, respectively, while ABI at 1-1.4 and > 1.4 are normal and non-compressible arteries (possibly from diabetes mellitus), respectively. ${ }^{31}$ The CAVI score $<8$ and 8-9 are normal and at risk for atherosclerosis, while CAVI $>9$ is possible atherosclerosis. ${ }^{29,30}$

\section{Statistical analysis}

Analyzed data using Statistical Package for Social Sciences software (SPSS 22.0, SPSS Inc., IL, USA) and Graph Pad Prism version 7.0 software (La Jolla, CA, USA) are presented as mean \pm standard error (SE) and mean \pm standard deviation (SD) for mouse and patient data, respectively. The differences between groups were examined for statistical significance by one-way analysis of variance (ANOVA) followed by Tukey's analysis or Student's t-test for comparisons of multiple or 2 groups, respectively. In parallel, Pearson correlation was used for the correlation coefficients and $p<0.05$ was considered statistically significant. $\mathrm{R}^{2}$

\section{Results}

Detection of TMAO reductase in blood was associated with gut leakage and systemic inflammation in the mouse models

Mouse models of indirect and direct intestinal damage using CKD and DSS models, respectively, did not demonstrate diarrhea (data not shown) similar to the previous publications. ${ }^{5,23}$ As such, renal injury (BUN and serum creatinine) was detectable in CKD mice but not in DSS mice (Figure 1A, B) with systemic inflammation (serum IL-6) in both models (more prominent in DSS than CKD mice) (Figure 1C). In parallel, gut leakage was demonstrated in both models with the higher severity in DSS mice than CKD mice as determined by FITC-dextran assay, endotoxemia, and the detection of Claudin-1, a tight junction molecule (Figure 1D-G). In addition, serum TMAO reductase was prominent in CKD mice and DSS mice (higher in DSS model) (Figure 1H) possibly due to mucositis-induced gut translocation. Meanwhile, TMAO, a uremic toxin, increased only in CKD mice but not in DSS mice (Figure 1I) which corresponded to 
A

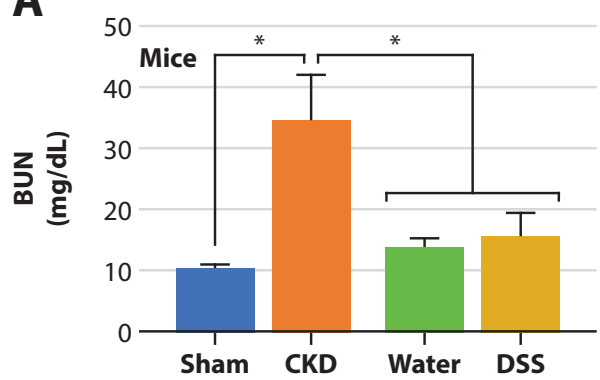

D

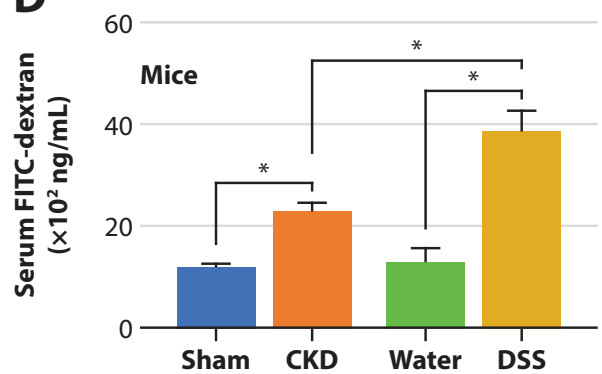

G
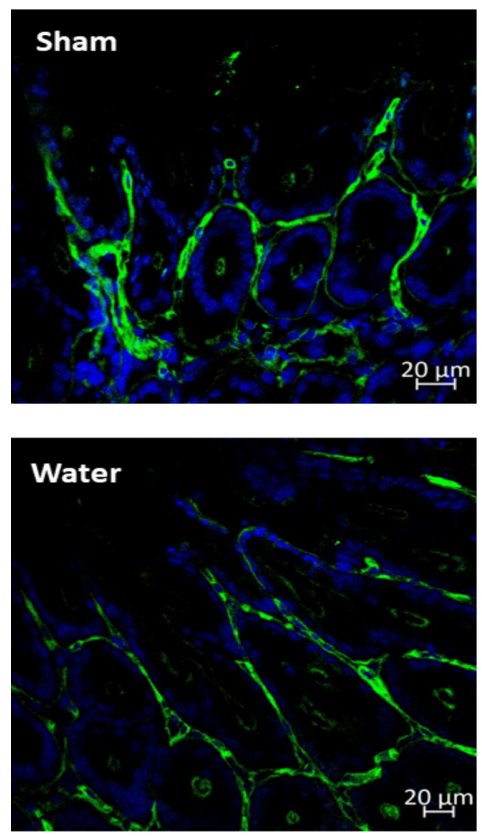

J

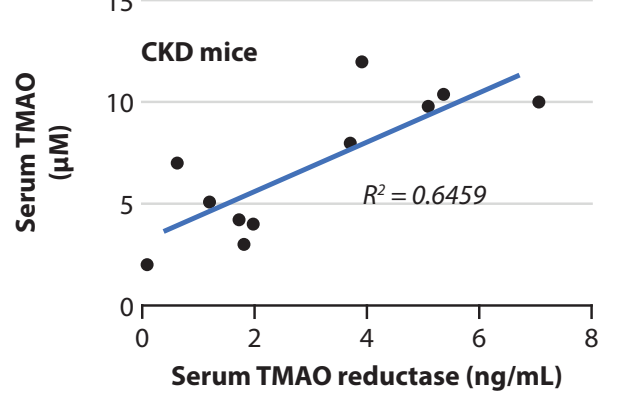

B

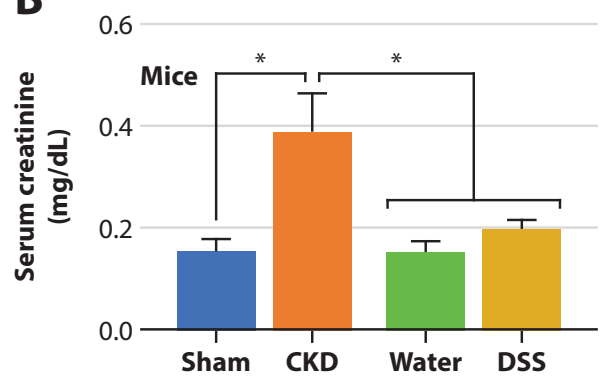

E
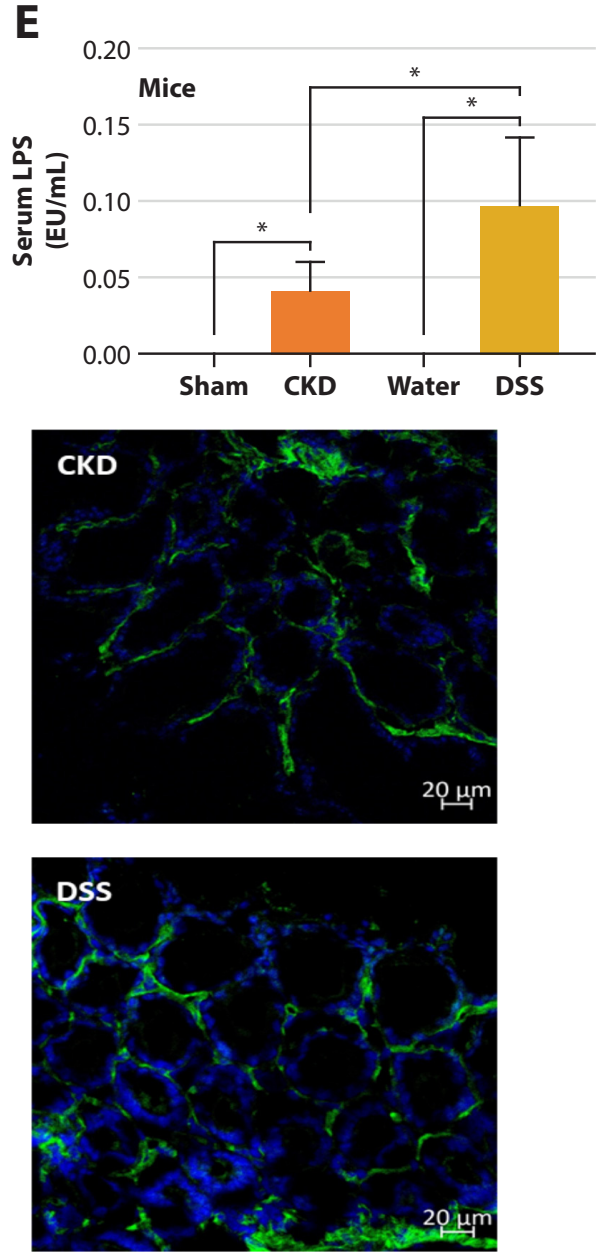

K

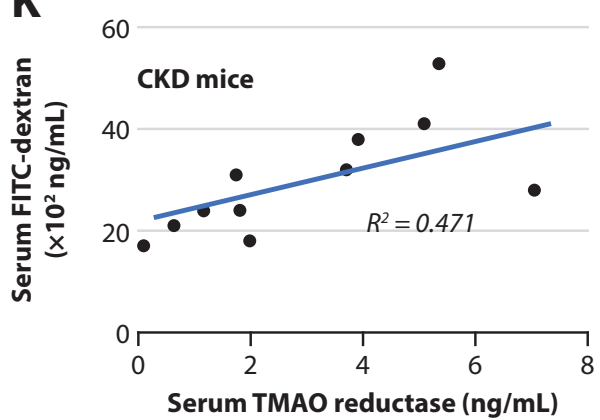

C

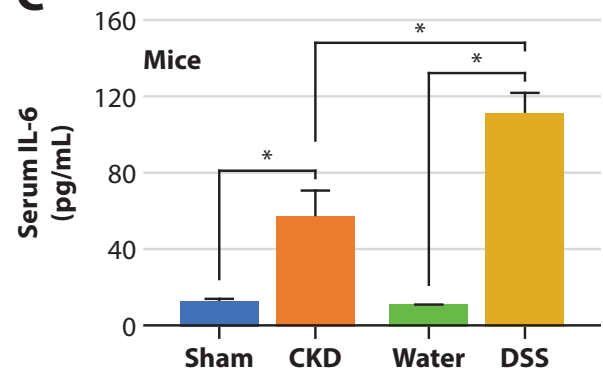

F

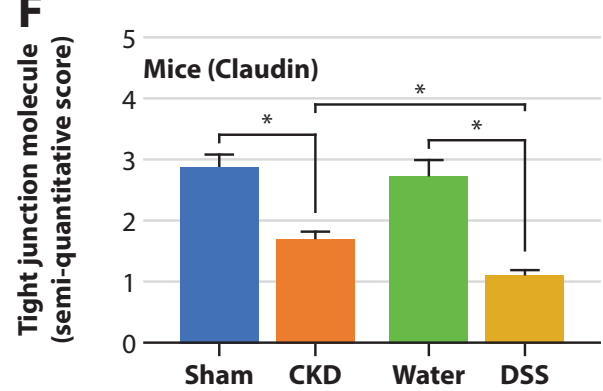

H

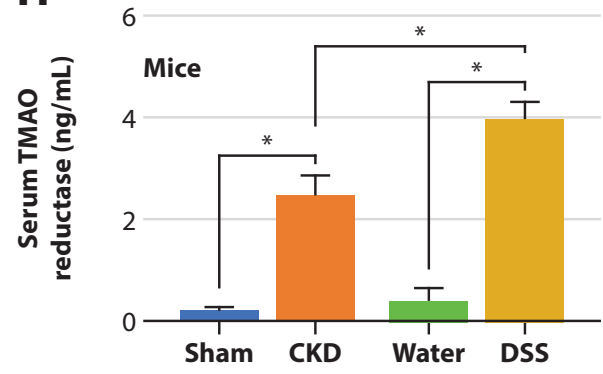

I

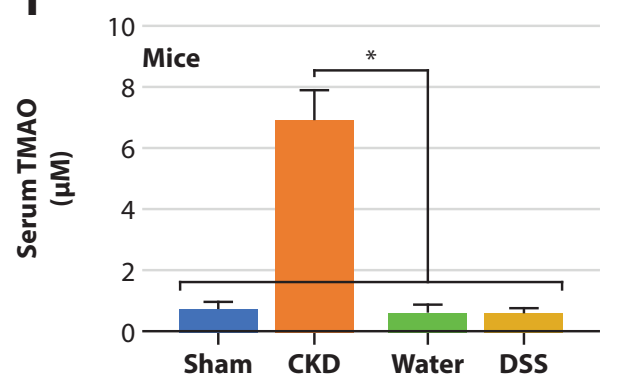

L

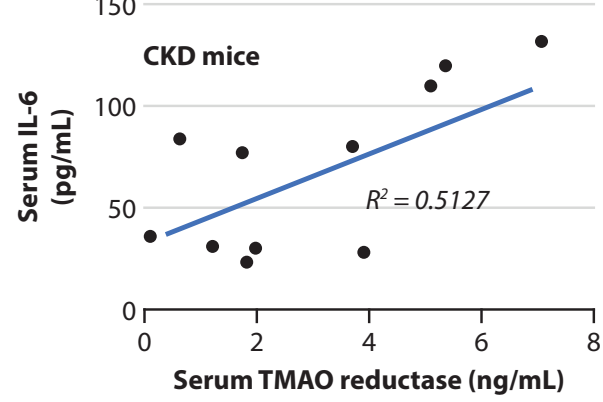

Figure 1. Characteristics of mice with chronic kidney disease (CKD), 3 months after 5/6 nephrectomy, or sham surgery and 1 week of $1.5 \%$ dextran sulfate solution (DSS) induced mucositis or drinking water control group as determined by i) renal function; blood urea nitrogen (BUN) and serum creatinine (A, B), ii) systemic inflammation (serum IL-6) (C), iii) gut permeability defect; serum FITC-dextran assay, endotoxemia, the immunofluorescent score of tight junction molecule (Claudin-1) and the representative figures (D-G), iv) serum TMAO reductase and serum TMAO (H, I) are demonstrated (n = 8-11/group). Additionally, the correlation between serum TMAO reductase against serum TMAO, FITC-dextran (gut leakage biomarker), or serum IL-6 (systemic inflammation) in the CKD model $(\mathrm{J}-\mathrm{L})$ is demonstrated. ${ }^{*} p<0.05$ 
the high TMAO reductase in CKD mice (Figure $\mathbf{1 H}$ ) possibly due to the enhanced reductase enzyme from gut bacteria to metabolite uremia-enhanced production of TMAO. ${ }^{32}$ As such, there was a good correlation between TMAO reductase and TMAO in CKD mice (Figure 1J).

Additionally, serum TMAO reductase (172 kDa MW) ${ }^{15}$ was associated with gut leakage using FITC-dextran $(4 \mathrm{kDa}$ MW) (Figure 1K) with $\mathrm{R}^{2}>0.4$ but was not correlated with LPS (50-100 kDa MW) ${ }^{2}$ (data not shown) possibly due to the higher MW of TMAO reductase. Moreover, serum TMAO reductase also correlated with systemic inflammation (serum IL-6) (Figure 1L) possibly due to the host reaction against molecules from gut leakage. ${ }^{33}$ Despite a previously mentioned uremia-induced enterocyte apoptosis in bilateral nephrectomy mice, ${ }^{25}$ the intestinal apoptotic cells in the colon of CKD 5/6 nephrectomy mice were non-detectable. Only the apoptosis in inflammatory cells at lamina propria of the colon in both CKD and DSS mice (more prominent in DSS mice) was demonstrated (Figure 2A, B).

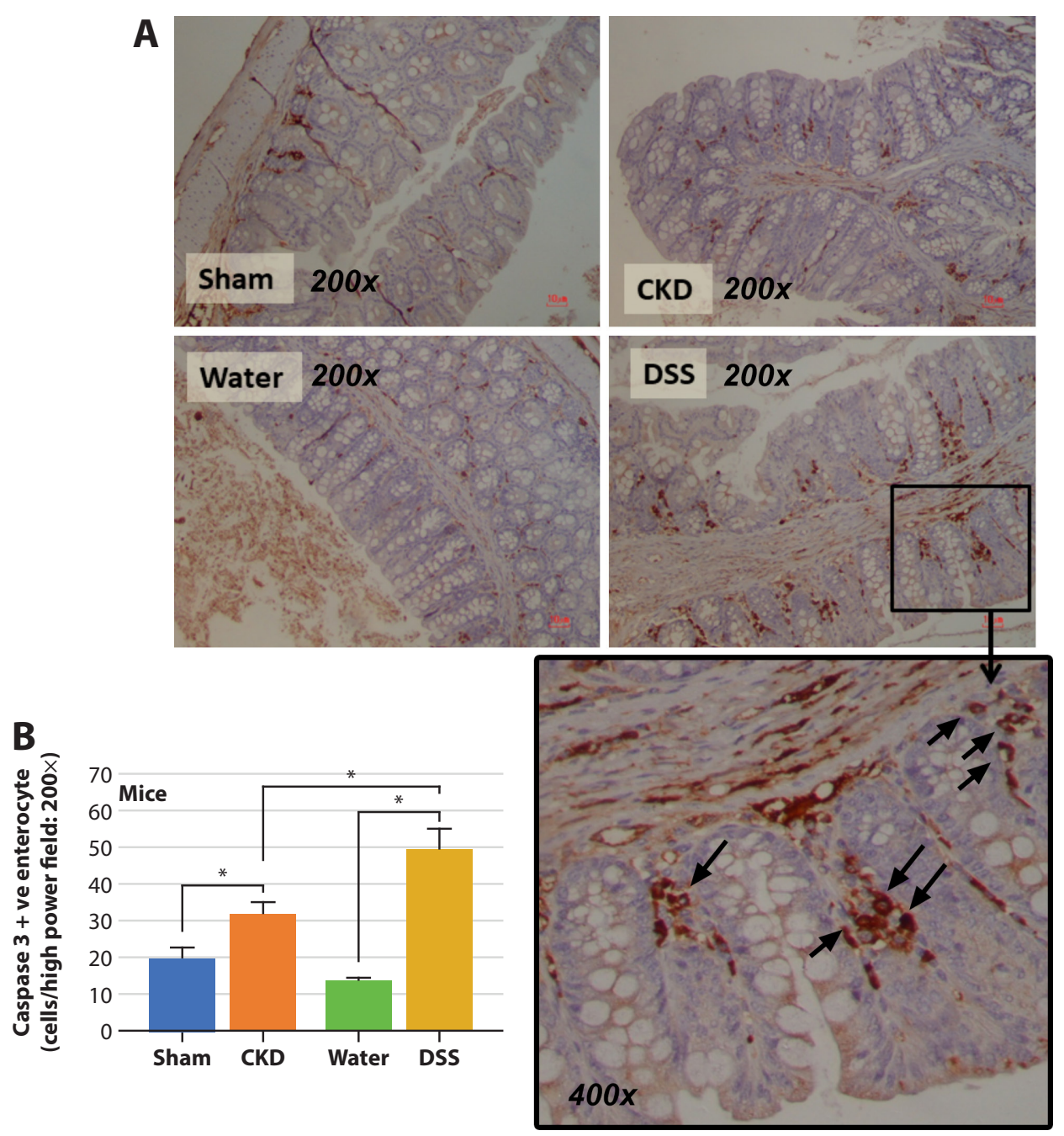

Figure 2. Detection of intestinal apoptotic cells in colons using caspase 3 immunohistochemistry staining from mice with chronic kidney disease (CKD; 3 months after 5/6 nephrectomy) or sham surgery and mice with 1 week of $1.5 \%$ dextran sulfate solution (DSS) induced mucositis or drinking water control group as indicated by the representative pictures $(200 \times$ for original magnification and $400 \times$ for the inset image) (A) and apoptotic cells abundance (cells/high power field at 200×) (n =6-8/group) (B). ${ }^{*} p<$ 0.05 ; The arrows indicate some of the positive apoptosis in mononuclear cells at the laminar propria (but not enterocytes) in the picture. 
Increased serum TMAO reductase in patients with chronic hemodialysis was not associated with endotoxemia and serum TMAO

The data from mice suggest that TMAO reductase might be used as a biomarker of gut permeability defect on CKD. For a proof of concept, a cross-sectional analysis of blood samples from patients was performed. Accordingly, blood samples from patients with chronic hemodialysis were analyzed and divided into patients with endotoxemia and non-endotoxemia as the demographic data presented in table 1. The liver function (SGOT and SGPT) of the patients was within the normal range $(<40 \mathrm{U} / \mathrm{L})$ despite the slightly higher level than the healthy control (Figure 3A, B). In parallel, all of the uremia toxins; small molecules (urea nitrogen and creatinine), a middle molecule $(\beta 2 \mathrm{M})$, and a protein-bound molecule (TMAO), in patients with CKD were higher than the healthy control (Figure 3C-F). Additionally, endotoxemia was detectable in 12 out of 48 patients (25\%), but not in the healthy control (Figure 3G), supporting the possible gut permeability defect. ${ }^{25}$ In parallel, serum TMAO reductase, a bacterial enzyme, was also higher in a patient with CKD together with inflammatory responses (CRP and serum cytokines) (Figure $\mathbf{3 H}-\mathbf{K}$ ). Overall, the uremic toxins (BUN and creatinine, $\beta 2 \mathrm{M}$ and TMAO), TMAO reductase, endotoxemia (in 12 out of 48 patients), CRP, and serum cytokines in patients with CKD were higher than the healthy control (Figure 3C-K). There was no correlation between TMAO and TMAO reductase. Also, both TMAO and TMAO reductase were not associated with other uremic toxins (creatinine and $\beta 2 \mathrm{M}$ ), systemic inflammation (serum IL-6) $\left(\mathrm{R}^{2}<0.4\right)($ Figure 4A-G), and LPS (Figure 4H-I)

Table 1. Characteristics of patients with or without endotoxemia.

\begin{tabular}{|c|c|c|}
\hline & $\begin{array}{l}\text { Endotoxemia } \\
\qquad(\mathrm{n}=12)\end{array}$ & $\begin{array}{l}\text { Non-endotoxemia } \\
\qquad(\mathrm{n}=36)\end{array}$ \\
\hline Age (mean \pm SD) (years) & $65 \pm 14$ & $64 \pm 11$ \\
\hline \multicolumn{3}{|l|}{ Gender, n (\%) } \\
\hline Male & $4(33)$ & $18(50)$ \\
\hline Female & $8(67)$ & $18(50)$ \\
\hline Dry weight (mean $\pm S D)(\mathrm{kg})$ & $58 \pm 14$ & $62 \pm 16$ \\
\hline Height $($ mean $\pm \mathrm{SD})(\mathrm{cm})$ & $159 \pm 7$ & $161 \pm 10$ \\
\hline $\mathrm{BMI}($ mean $\pm \mathrm{SD})$ & $23 \pm 5$ & $24 \pm 5$ \\
\hline Dialysis vintage (mean $\pm \mathrm{SD}$ ) (years) & $12 \pm 5$ & $12 \pm 5$ \\
\hline \multicolumn{3}{|l|}{ Primary kidney disease, n (\%) } \\
\hline Diabetic nephropathy & $4(33)$ & $20(55)$ \\
\hline Hypertension & $4(33)$ & $4(11)$ \\
\hline Glomerulonephritis & $1(8)$ & $3(8)$ \\
\hline Unknown & $3(25)$ & $9(25)$ \\
\hline \multicolumn{3}{|l|}{ Laboratory $($ mean \pm SD) } \\
\hline Fasting plasma glucose (FBS) (mg/dL) & $104 \pm 31$ & $107 \pm 27$ \\
\hline SGOT (U/L) & $17 \pm 8$ & $20 \pm 7$ \\
\hline SGPT (U/L) & $16 \pm 10$ & $18 \pm 8$ \\
\hline BUN (mg/dL) & $66 \pm 26$ & $68 \pm 20$ \\
\hline Creatinine (mg/dL) & $8 \pm 3$ & $9 \pm 3$ \\
\hline Beta-2 microglobulin (ug/L) & $31,877 \pm 8,985$ & $28,963 \pm 7,346$ \\
\hline Serum C-RP $(\mathrm{mg} / \mathrm{L})^{*}$ & $8 \pm 11$ & $4 \pm 10$ \\
\hline Serum TMAO (uM) & $135 \pm 162$ & $110 \pm 140$ \\
\hline Serum TMAO reductase (ng/dL) & $14 \pm 5$ & $16 \pm 15$ \\
\hline Serum TNF-alpha (pg/mL) & $154 \pm 26$ & $154 \pm 530$ \\
\hline Serum IL-6 (pg/mL) & $4 \pm 4$ & $24 \pm 52$ \\
\hline Serum LPS (EU/mL) & $0.04 \pm 0.07$ & $<0.01$ \\
\hline
\end{tabular}

${ }^{*} p<0.05$ between groups 
A

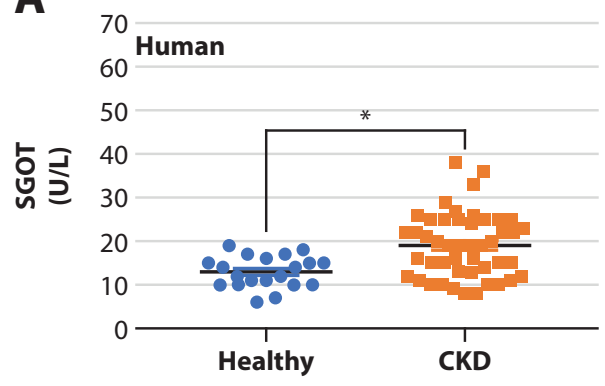

D

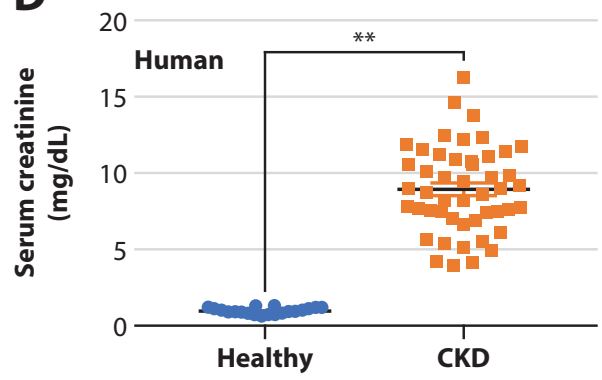

G

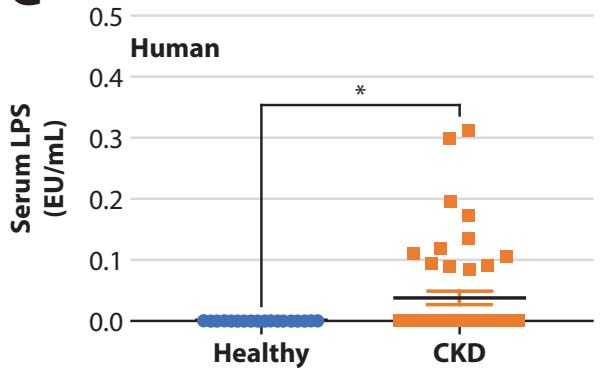

B

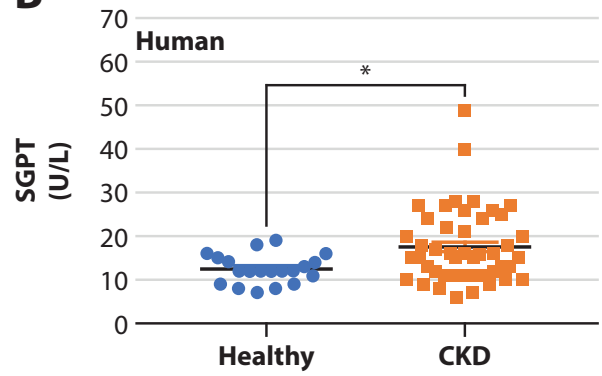

E

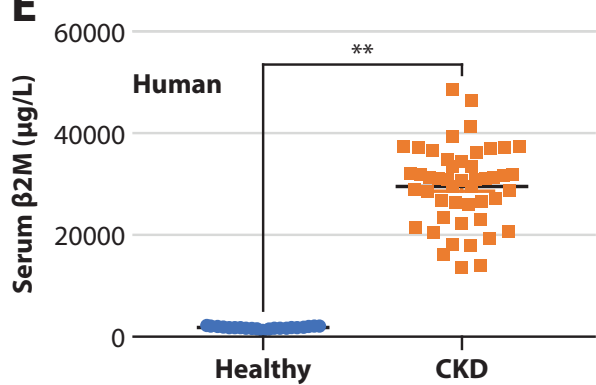

H

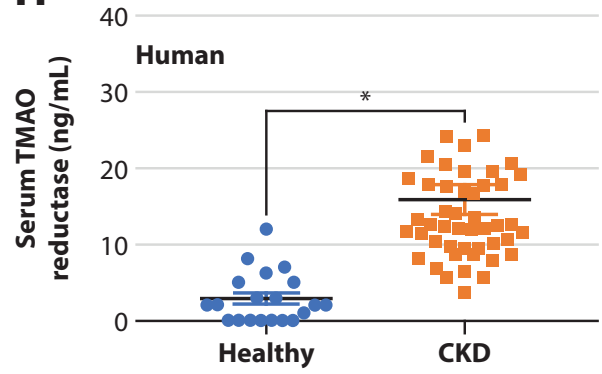

C

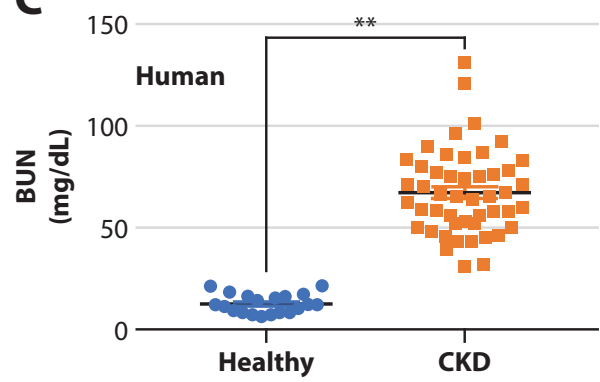

F

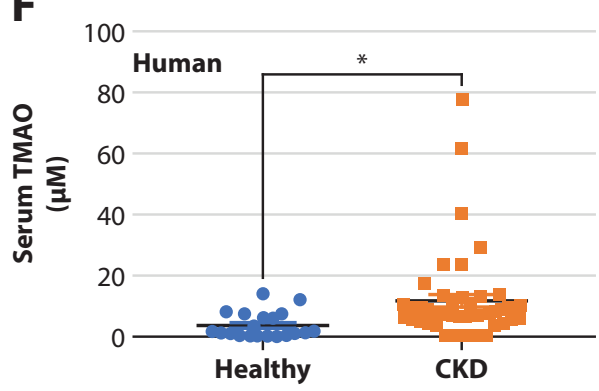

I

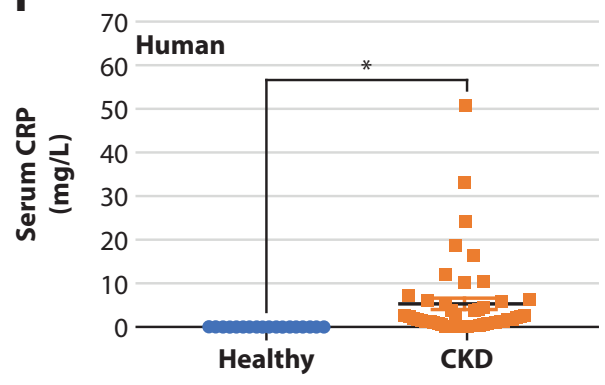

J

K
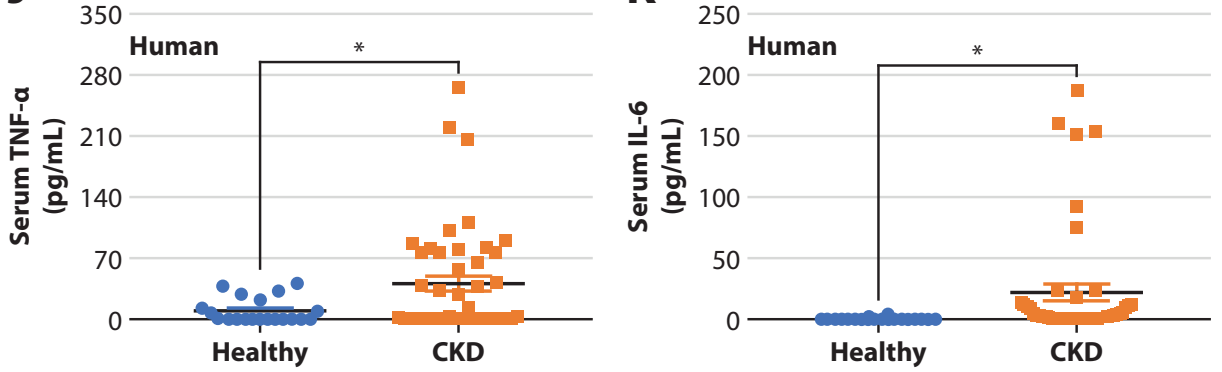

Figure 3. Characteristics of the healthy volunteers $(n=20)$ and the patients with chronic kidney disease (CKD) (see method) ( $\mathrm{n}$ $=48$ ) as determined by i) liver injury; serum glutamic-oxaloacetic transaminase (SGOT) and serum glutamate-pyruvate transaminase (SGPT) (A, B), ii) uremic toxins of small molecules; blood urea nitrogen (BUN) and serum creatinine (C, D), middle molecule; beta-2 microglobulin $(\beta 2 \mathrm{M})(\mathrm{E})$, protein-bound uremic toxin; Trimethylamine N-oxide (TMAO) (F), iii) gut leakage (endotoxemia) (G), iv) TMAO reductase (H) and v) inflammatory responses; C-reactive protein (CRP), serum TNF- $\alpha$ and IL-6 $(\mathrm{I}-\mathrm{K})$ are demonstrated. ${ }^{\star} p<0.05 ;{ }^{* *} p<0.001$ 


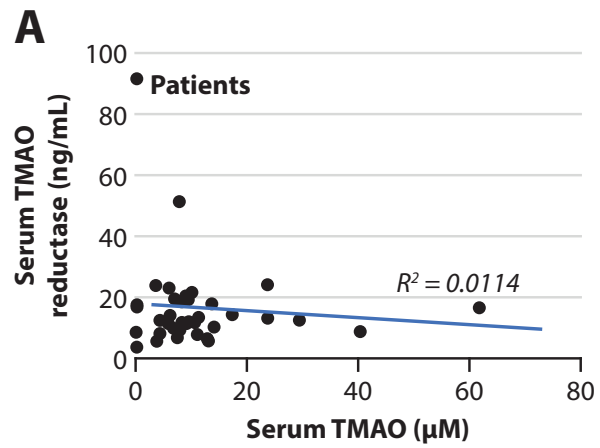

B

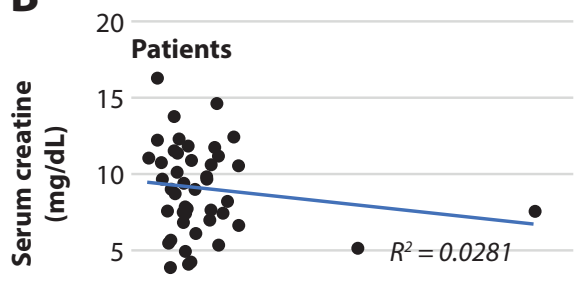

D
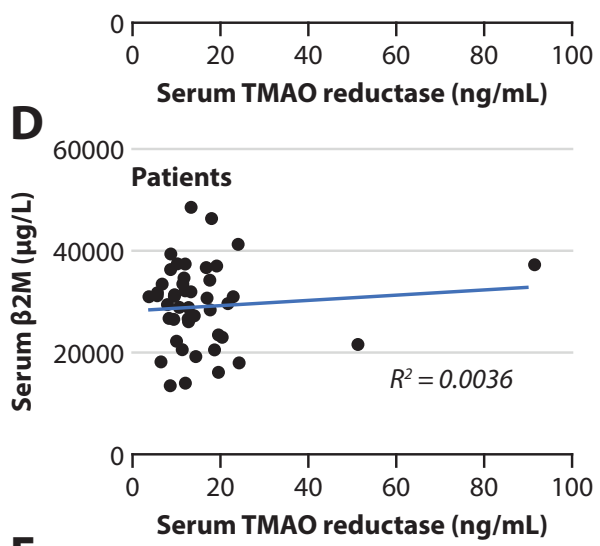

F

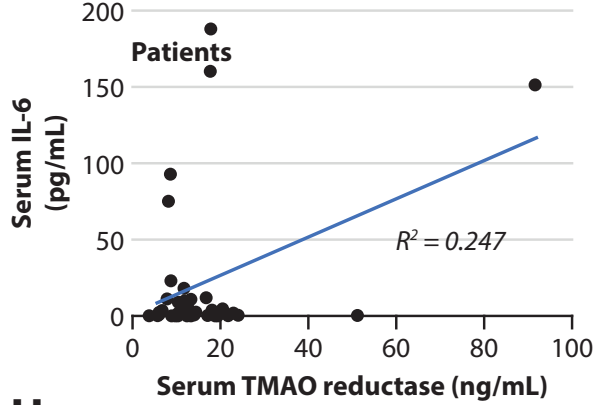

H

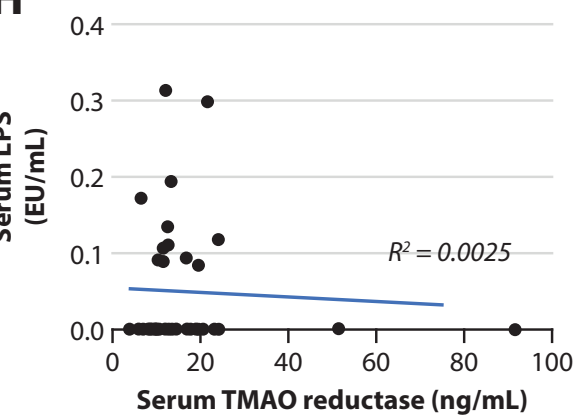

C

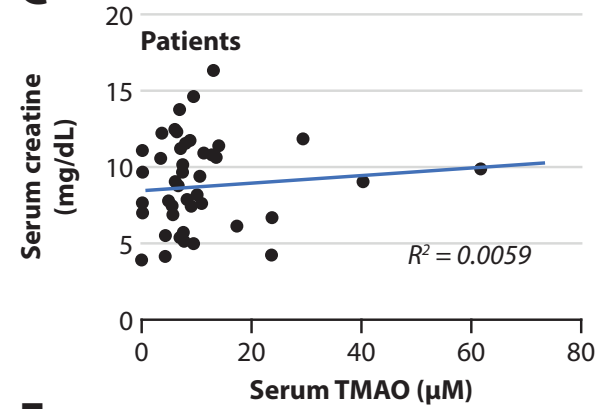

E 60000
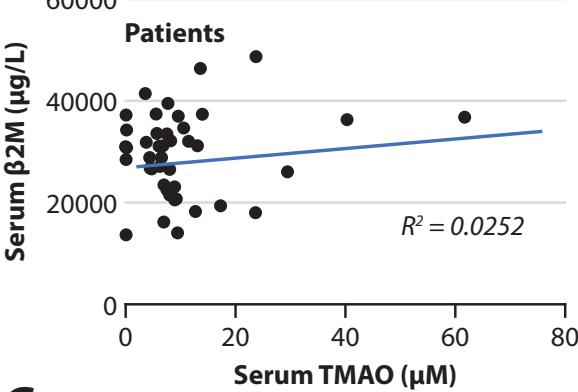

G

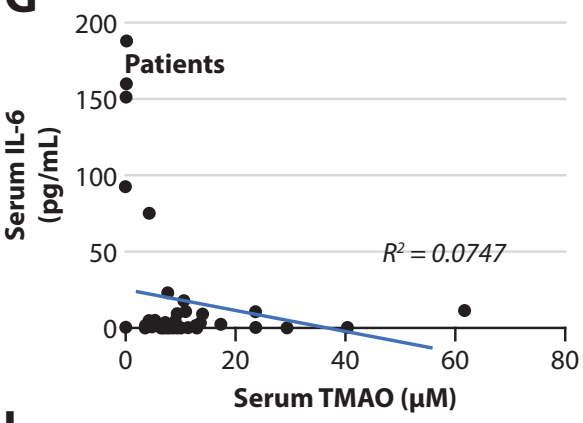

I

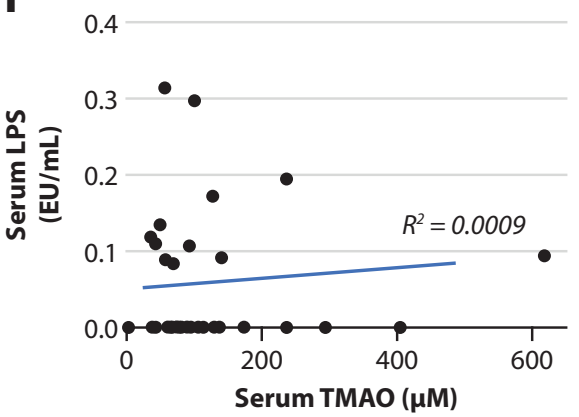

Figure 4. The correlation between serum Trimethylamine N-oxide (TMAO) reductase and serum TMAO (A) and between serum TMAO reductase or serum TMAO with serum creatinine (a small molecule uremic toxin) (B, C), beta-2 microglobulin ( $\beta 2 \mathrm{M})(\mathrm{a}$ middle molecule uremic toxin) (D, E) or systemic inflammation (serum IL-6) (F, G) and endotoxemia (H, I) from patients with chronic kidney disease $(\mathrm{CKD})$ (see method) $(\mathrm{n}=48)$ are demonstrated. 
A

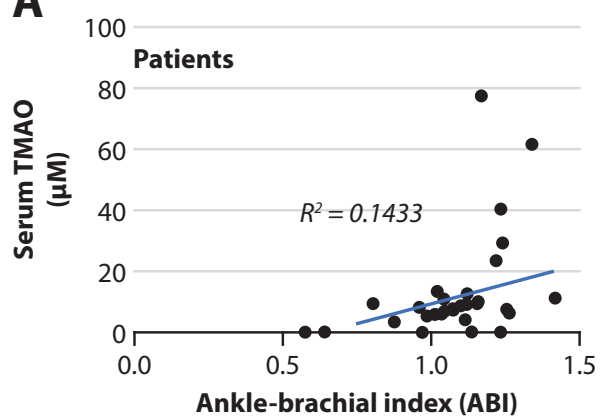

D

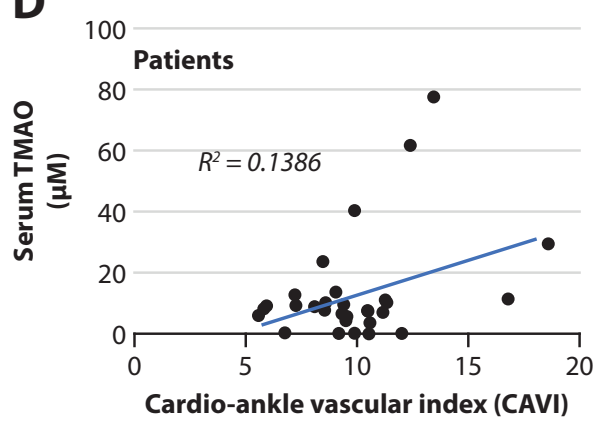

G

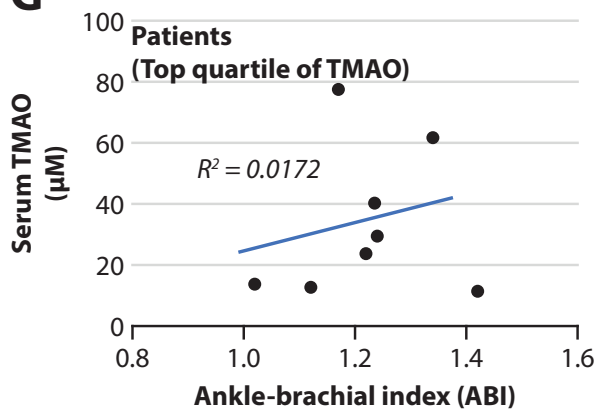

J

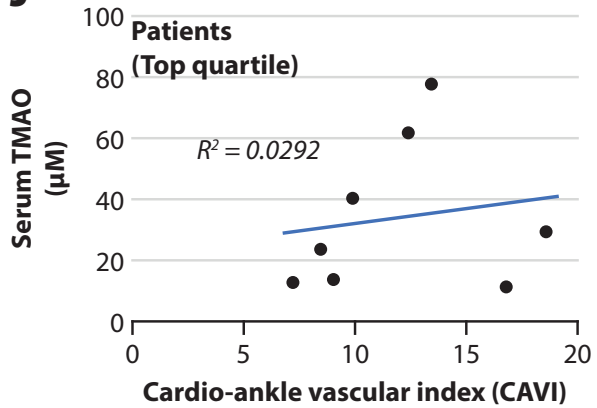

B

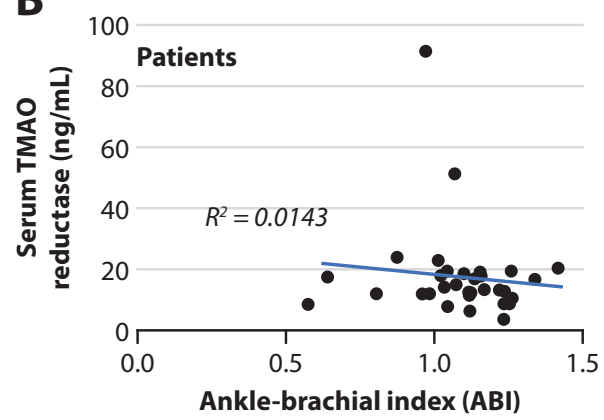

$\mathbf{E}$

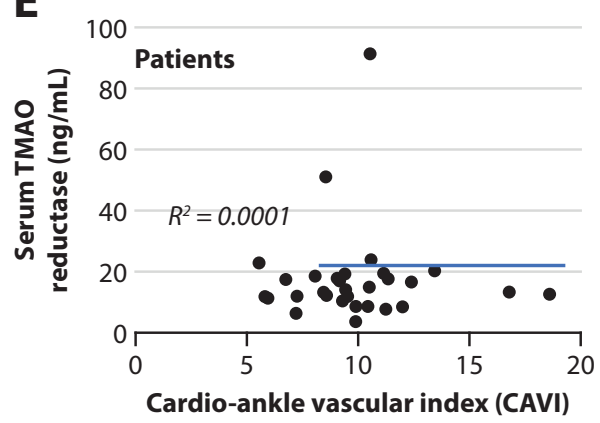

H

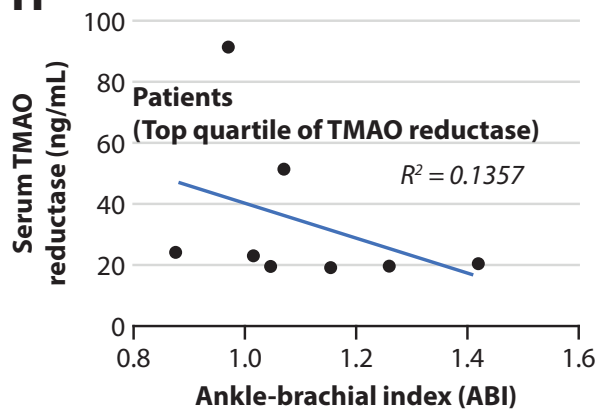

K

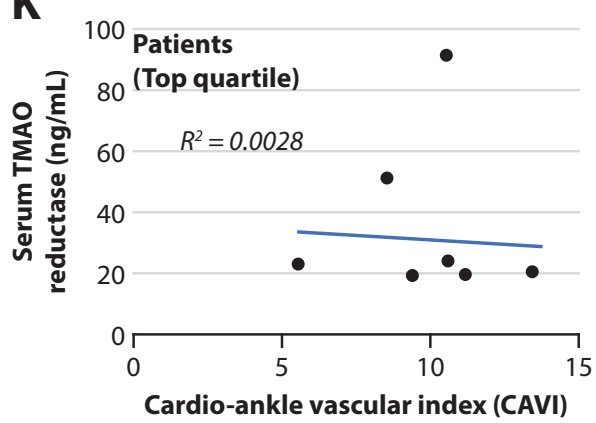

C

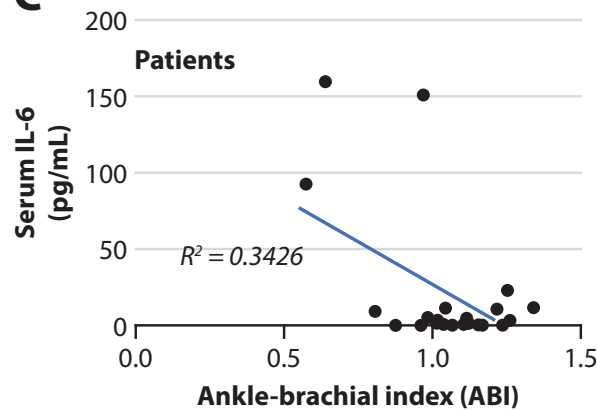

$\mathbf{F}$

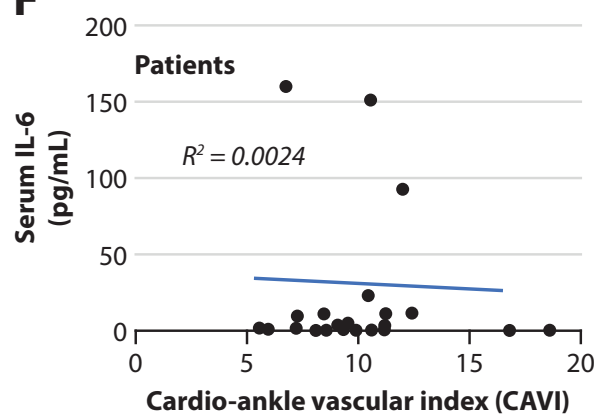

I

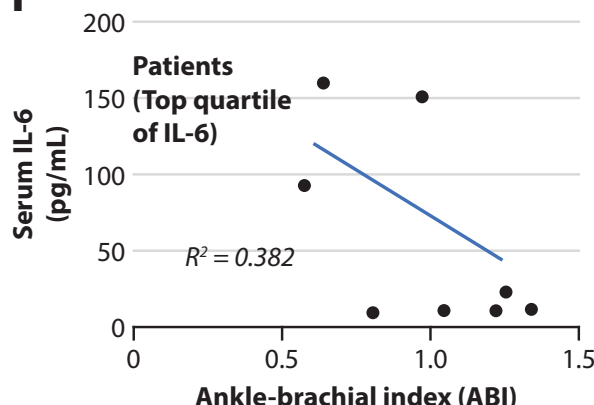

$\mathbf{L}$

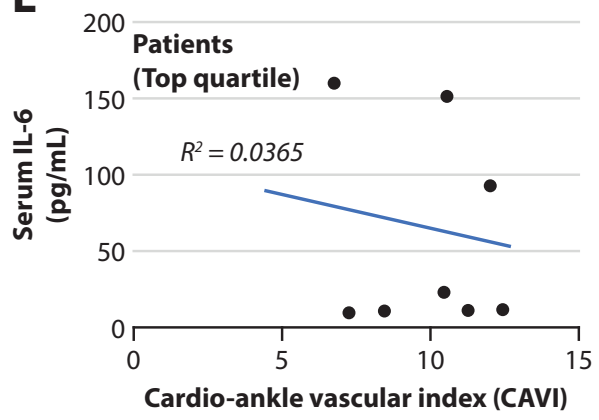

Figure 5. The correlation between serum Trimethylamine N-oxide reductase (TMAO; a protein-bound uremic toxin), serum TMAO reductase, or systemic inflammation (serum IL-6) versus ankle-brachial index (ABI) (A-C) or cardio ankle vascular index $(\mathrm{CAVI})$, the indicators of atherosclerosis $(\mathrm{D}-\mathrm{F})(\mathrm{n}=32)$, are demonstrated. Additionally, the correlation between the top quartile values of TMAO, TMAO reductase, or IL-6 in serum versus ABI or CAVI $(\mathrm{G}-\mathrm{L})(\mathrm{n}=8)$ is also demonstrated.

suggesting a lesser impact of TMAO reductase in patients with CKD. Because of the well-known correlation between serum TMAO and CKD vascular complications, ${ }^{16-18}$ ankle-brachial index (ABI) and cardio-ankle vascular index (CAVI), the indicators of peripheral arterial disease and arterial stiffness, respectively, were measured in patients. Unfortunately, there was no correlation between serum TMAO, TMAO reductase, or serum IL-6 with neither ABI nor CAVI (Figure 5A-F). Notably, only serum IL-6 demonstrated the negative correlation to $\mathrm{ABI}$ (the lower $\mathrm{ABI}$ values, the more severe arterial stiffness) (Figure 5C), there was still no correlation to $\mathrm{ABI}$ and CAVI score with the highest quartile of these values (Figure 5G-L). Hence, increased TMAO reductase in patients with CKD might be slightly associated with systemic inflammation (Figure 4F), but not correlated with TMAO (Figure 4A) and the arterial complications (Figure 5B, E). The $p$ values from Figure 4 and Figure 5 are demonstrated in Supplement Table 1. 


\section{Discussion}

Increased serum TMAO reductase in mouse models of gut leakage by uremia and DSS mucositis

The reduced toxin elimination through the kidney in CKD might enhance the excretion of trimethylamine $\mathrm{N}$-oxide (TMAO), the uremic toxin formed in the liver, into the intestine that possibly induced TMAO reductase (a TMAO-neutralizing enzyme) in gut bacteria. The hypothesis that "the detection of TMAO reductase in serum might be used as a biomarker of gut permeability defect" was tested. The intestinal damage in 5/6 nephrectomy-induced CKD (an indirect intestinal injury from uremia) and DSS mucositis model (a direct gut injury model) was demonstrated. Accordingly, the intestinal permeability defect in the DSS model was more severe than the CKD model as indicated by the higher FITC-dextran and LPS with the lower abundance of a tight junction molecule (Claudin-1) supporting the previous publications. ${ }^{21,25}$ Due to gut translocation of LPS (MW 50-100 $\mathrm{kDa}$ ), the damage of intestinal mucosa in both models was severe enough for gut translocation of microbial molecules with high molecular weight. ${ }^{1}$ Perhaps, the presentation of TMAO reductase (MW $172 \mathrm{kDa}$ ) in serum of these mice might also due to gut permeability defect. Although enterocyte apoptosis in gut mucosa allows gut translocation of the larger microbial molecules (or the whole viable organisms), ${ }^{1}$ apoptosis of the mononuclear cells, but not enterocytes, were detectable at lamina propria of both CKD and DSS model. These accumulated lymphoid cells with apoptosis may be also responsible for the enhanced intestinal inflammation. ${ }^{34}$ However, serum TMAO was detectable only in CKD mice, but not DSS mice, supporting the property of TMAO as a uremic toxin. Despite fecal dysbiosis in both uremia ${ }^{25}$ and DSS, ${ }^{21}$ the dysbiosis in uremia (but not DSS) enhanced TMAO suggesting the different selected growth of some bacteria between these models. Surprisingly, TMAO and TMAO reductase in serum of DSS mice were higher and lower than CKD mice, respectively, implying the enhanced TMAO reductase production by other non-TMAO factors in DSS mice. Indeed, TMAO reductase in Vibrio fluvialis could be inducible by the high hydrostatic pressure. ${ }^{35}$ Hence, some stress factors in the gut microenvironment in the DSS colitis model might induce TMAO reductase. More studies are needed. On the other hand, in our attempts to detect the level in fecal contents, fecal TMAO reductase in both DSS and CKD models were still higher than the upper range of the standard curve of the assay which possibly due to the extremely high level of TMAO reductase or the non-specific binding to other substances in the fecal contents (data not shown). More studies on these topics are interesting. Nevertheless, the correlation between TMAO and TMAO reductase in the CKD mouse model implied a possibility to use TMAO reductase as a CKD biomarker.
Serum TMAO reductase increased in patients with CKD but did not correlate with serum TMAO, discordance between the CKD mouse model and the patients

Because blood collection was performed before hemodialysis without clinical infection, endotoxemia in these patients did not dialysis contamination but most likely from gut permeability defect. There was slightly increased CRP $(8 \pm 3$ $\mathrm{mg} / \mathrm{L}$; normal value $<5 \mathrm{mg} / \mathrm{L}$ ) in patients with endotoxemia, despite the non-elevation of serum cytokines. However, serum LPS was non-detectable on the repeated measurement at 1 week later in all of these patients (data not shown) implying transient endotoxemia possibly from diet or other factors. More studies are interesting.

Different from the CKD mice, TMAO reductase in patients with CKD did not correlate with LPS (a marker of possible gut leakage), TMAO, inflammatory cytokines, and biomarkers of vascular complication (ABI and CAVI) implied a limitation of TMAO reductase in the real clinical situation. Despite an increase in TMAO reductase in patients with CKD, the benefit of TMAO reductase over other CKD biomarkers is limited. As such, the discordance between patients and mice might due to the limitation of mouse models or the difference in several clinical patient factors; including medications, underlying diseases, and hemodialysis. Notably, the non-correlation between serum TMAO and vascular complications (ABI and CAVI) different from most of the publications on the topic ${ }^{16-18}$ implied a limitation in the number of patients in our pilot study. The studies on a proper number of patients with end-stage renal disease (ESRD) before hemodialysis with direct measurement of gut leakage are needed.

In conclusion, increased TMAO reductase in CKD mice was correlated with serum TMAO, gut leakage, and systemic inflammation. However, TMAO reductase in patients with CKD did not correlate with serum TMAO, endotoxemia (a surrogate marker of gut leakage), and systemic inflammation, despite the increased serum level in patients compared with the healthy control, demonstrated a limitation of TMAO reductase in the hemodialysis patients. More studies in patients with ESRD without hemodialysis are interesting.

\section{Conflicts of interest \\ None}

\section{Funding}

This study was supported by Thailand Research Fund (RES_61_202_30_022), Ratchadapisek Sompoch (CU_GR_63 _108_3) and the Program Management Unit for Human Resources \& Institutional Development Research and Innovation-CU [Global Partnership B16F630071 and Flagship B05F630073] and National Research Council of Thailand. 


\section{References}

1. Amornphimoltham P, Yuen PST, Star RA, Leelahavanichkul A. Gut Leakage of Fungal-Derived Inflammatory Mediators: Part of a Gut-Liver-Kidney Axis in Bacterial Sepsis. Dig Dis Sci. 2019;64(9):2416-28.

2. Panpetch W, Sawaswong V, Chanchaem P, Ondee T, Dang CP, Payungporn $S$, et al. Corrigendum: Candida Administration Worsens Cecal Ligation and Puncture-Induced Sepsis in Obese Mice Through Gut Dysbiosis Enhanced Systemic Inflammation, Impact of Pathogen-Associated Molecules From Gut Translocation and Saturated Fatty Acid. Front Immunol. 2020;11:613095.

3. Bhunyakarnjanarat T, Udompornpitak K, Saisorn W, Chantraprapawat B, Visitchanakun P, Dang CP, et al. Prominent Indomethacin-Induced Enteropathy in Fcgriib Defi-cient lupus Mice: An Impact of Macrophage Responses and Immune Deposition in Gut. Int J Mol Sci. 2021;22(3).

4. Thim-Uam A, Surawut S, Issara-Amphorn J, Jaroonwitchawan T, Hiengrach P, Chatthanathon P, et al. Leaky-gut enhanced lupus progression in the Fc gamma receptor-IIb deficient and pristane-induced mouse models of lupus. Sci Rep. 2020;10(1):777.

5. Panpetch W, Somboonna N, Bulan DE, Issara-Amphorn J, Finkelman M, Worasilchai N, et al. Oral administration of live- or heat-killed Candida albicans worsened cecal ligation and puncture sepsis in a murine model possibly due to an increased serum (1-->3)-beta-D-glucan. PLoS One. 2017;12(7):e0181439.

6. Issara-Amphorn J, Surawut S, Worasilchai N, Thim-Uam A, Finkelman M, Chindamporn A, et al. The Synergy of Endotoxin and (1-->3)-beta-D -Glucan, from Gut Translocation, Worsens Sepsis Severity in a Lupus Model of Fc Gamma Receptor IIb-Deficient Mice. J Innate Immun. 2018;10(3):189-201.

7. Mohammad S, Thiemermann C. Role of Metabolic Endotoxemia in Systemic Inflammation and Potential Interventions. Front Immunol. 2020;11:594150.

8. Camilleri M. Leaky gut: mechanisms, measurement and clinical implications in humans. Gut. 2019;68(8):1516-26.

9. Chancharoenthana W, Wattanatorn S, Vadcharavivad S, Eiam-Ong S, Leelahavanichkul A. Agreement and Precision Analyses of Various Estimated Glomerular Filtration Rate Formulae in Cancer Patients. Sci Rep. 2019;9(1):19356.

10. Wang F, Zhang $\mathrm{P}$, Jiang $\mathrm{H}$, Cheng $\mathrm{S}$. Gut bacterial translocation contributes to microinflammation in experimental uremia. Dig Dis Sci. 2012;57(11):2856-62.

11. Vaziri ND, Goshtasbi N, Yuan J, Jellbauer S, Moradi H, Raffatellu M, et al. Uremic plasma impairs barrier function and depletes the tight junction protein constituents of intestinal epithelium. Am J Nephrol. 2012;36(5): 438-43.

12. Rydzewska-Rosolowska A, Sroka N, Kakareko K, Rosolowski M, Zbroch E, Hryszko T. The Links between Microbiome and Uremic Toxins in Acute Kidney Injury: Beyond Gut Feeling-A Systematic Review. Toxins (Basel). 2020;12(12).

13. Tang WH, Wang Z, Kennedy DJ, Wu Y, Buffa JA, Agatisa-Boyle B, et al. Gut microbiota-dependent trimethylamine N-oxide (TMAO) pathway contributes to both development of renal insufficiency and mortality risk in chronic kidney disease. Circ Res. 2015;116(3):448-55.

14. Barrett EL, Kwan HS. Bacterial reduction of trimethylamine oxide. Annu Rev Microbiol. 1985;39:131-49.

15. Arata H, Shimizu M, Takamiya K. Purification and properties of trimethylamine $\mathrm{N}$-oxide reductase from aerobic photosynthetic bacterium Roseobacter denitrificans. J Biochem. 1992;112(4):470-5.

16. Roncal C, Martinez-Aguilar E, Orbe J, Ravassa S, Fernandez-Montero A, Saenz-Pipaon G, et al. Trimethylamine-N-Oxide (TMAO) Predicts Cardiovascular Mortality in Peripheral Artery Disease. Sci Rep. 2019;9(1): 15580.

17. Stubbs JR, House JA, Ocque AJ, Zhang S, Johnson C, Kimber C, et al. Serum Trimethylamine-N-Oxide is Elevated in CKD and Correlates with Coronary Atherosclerosis Burden. J Am Soc Nephrol. 2016;27(1):305-13.

18. Hsu CN, Chang-Chien GP, Lin S, Hou CY, Lu PC, Tain YL. Association of Trimethylamine, Trimethylamine $\mathrm{N}$-oxide, and Dimethylamine with Cardiovascular Risk in Children with Chronic Kidney Disease. J Clin Med. 2020;9(2).
19. Leelahavanichkul A, Huang Y, Hu X, Zhou H, Tsuji T, Chen R, et al. Chronic kidney disease worsens sepsis and sepsis-induced acute kidney injury by releasing High Mobility Group Box Protein-1. Kidney Int. 2011;80(11): 1198-211.

20. Leelahavanichkul A, Yan Q, Hu X, Eisner C, Huang Y, Chen R, et al. Angiotensin II overcomes strain-dependent resistance of rapid CKD progression in a new remnant kidney mouse model. Kidney Int. 2010; 78(11):1136-53.

21. Panpetch W, Hiengrach P, Nilgate S, Tumwasorn S, Somboonna N, Wilantho A, et al. Additional Candida albicans administration enhances the severity of dextran sulfate solution induced colitis mouse model through leaky gut-enhanced systemic inflammation and gut-dysbiosis but attenuated by Lactobacillus rhamnosus L34. Gut Microbes. 2020;11(3): 465-80.

22. Hiengrach P, Panpetch W, Worasilchai N, Chindamporn A, Tumwasorn S Jaroonwitchawan T, et al. Administration of Candida Albicans to Dextran Sulfate Solution Treated Mice Causes Intestinal Dysbiosis, Emergence and Dissemination of Intestinal Pseudomonas Aeruginosa and Lethal Sepsis. Shock. 2020;53(2):189-98.

23. Leelahavanichkul A, Worasilchai N, Wannalerdsakun S, Jutivorakool K, Somparn P, Issara-Amphorn J, et al. Gastrointestinal Leakage Detected by Serum (1-->3)-beta-D-Glucan in Mouse Models and a Pilot Study in Patients with Sepsis. Shock. 2016;46(5):506-18.

24. Visitchanakun P, Saisorn W, Wongphoom J, Chatthanathon P, Som boonna N, Svasti S, et al. Gut leakage enhances sepsis susceptibility in iron-overloaded beta-thalassemia mice through macrophage hyperinflammatory responses. Am J Physiol Gastrointest Liver Physiol. 2020;318(5):G966-G79.

25. Panpetch W, Kullapanich C, Dang CP, Visitchanakun P, Saisorn W, Wongphoom J, et al. Candida Administration Worsens Uremia-Induced Gut Leakage in Bilateral Nephrectomy Mice, an Impact of Gut Fungi and Organismal Molecules in Uremia. mSystems. 2021;6(1)

26. Sae-Khow K, Charoensappakit A, Visitchanakun P, Saisorn W, Svasti S, Fucharoen S, et al. Pathogen-Associated Molecules from Gut Translocation Enhance Severity of Cecal Ligation and Puncture Sepsis in Iron-Overload beta-Thalassemia Mice. J Inflamm Res. 2020;13:719-35.

27. Gessner A, di Giuseppe R, Koch M, Fromm MF, Lieb W, Maas R. Trimethylamine-N-oxide (TMAO) determined by LC-MS/MS: distribution and correlates in the population-based PopGen cohort. Clin Chem Lab Med. 2020;58(5):733-40.

28. Levison BS, Zhang $\mathrm{R}$, Wang $\mathrm{Z}$, Fu X, DiDonato JA, Hazen SL. Quantification of fatty acid oxidation products using online high -performance liquid chromatography tandem mass spectrometry. Free Radic Biol Med. 2013;59:2-13.

29. Asmar R, Sonett JR, Singh G, Mansukhani MM, Borczuk AC. Use of Oncogenic Driver Mutations in Staging of Multiple Primary Lung Carcinomas: A Single-Center Experience. J Thorac Oncol. 2017;12(10): 1524-35.

30. Takahashi K, Yamamoto T, Tsuda S, Maruyama M, Shirai K. The Background of Calculating CAVI: Lesson from the Discrepancy Between CAVI and CAVI0. Vasc Health Risk Manag. 2020;16:193-201.

31. Aboyans V, Criqui MH, Abraham P, Allison MA, Creager MA, Diehm $\mathrm{C}$, et al. Measurement and interpretation of the ankle-brachial index: a scientific statement from the American Heart Association. Circulation. 2012;126(24):2890-909.

32. Shafi T, Powe NR, Meyer TW, Hwang S, Hai X, Melamed ML, et al. Trimethylamine N-Oxide and Cardiovascular Events in Hemodialysis Patients. J Am Soc Nephrol. 2017;28(1):321-31.

33. Dos Santos JP, Iobbi-Nivol C, Couillault C, Giordano G, Mejean V. Molecular analysis of the trimethylamine $\mathrm{N}$-oxide (TMAO) reductase respiratory system from a Shewanella species. J Mol Biol. 1998;284(2): 421-33.

34. Glorieux G, Gryp T, Perna A. Gut-Derived Metabolites and Their Role in Immune Dysfunction in Chronic Kidney Disease. Toxins (Basel). $2020 ; 12(4)$

35. Yin QJ, Zhang WJ, Qi XQ, Zhang SD, Jiang T, Li XG, et al. High Hydrostatic Pressure Inducible Trimethylamine N-Oxide Reductase Improves the Pressure Tolerance of Piezosensitive Bacteria Vibrio fluvialis. Front Microbiol. 2018;8:2646. 
Supplement table 1. The correlation and $p$ value from figure 4 and 5.

\begin{tabular}{|lcc|}
\hline \multicolumn{1}{|c}{ Groups of interest } & $\mathbf{R}^{2}$ & $p$ value \\
\hline TMAO reductase vs. TMAO & 0.0114 & 0.485 \\
\hline Creatinine vs TMAO reductase & 0.0281 & 0.271 \\
\hline Creatinine vs TMAO & 0.0059 & 0.616 \\
\hline B2M vs TMAO reductase & 0.0252 & 0.298 \\
\hline IL-6 vs TMAO reductase & 0.247 & 0.050 \\
\hline IL-6 vs TMAO & 0.0747 & 0.069 \\
\hline LPS vs TMAO reductase & 0.0025 & 0.744 \\
\hline LPS vs TMAO & 0.0009 & 0.845 \\
\hline TMAO vs. ankle brachial index (ABI) (all samples) & 0.1433 & 0.0527 \\
\hline TMAO reductase vs. ankle brachial index (ABI) (all samples) & 0.0143 & 0.515 \\
\hline IL-6 vs. ankle brachial index (ABI) (all samples) & 0.3426 & 0.0504 \\
\hline TMAO vs. cardio-ankle vascular index (CAVI) (all samples) & 0.1386 & 0.0516 \\
\hline TMAO reductase vs. Cardio-ankle vascular index (CAVI) (all samples) & 0.0001 & 0.957 \\
\hline IL-6 vs. cardio-ankle vascular index (CAVI) (all samples) & 0.0024 & 0.79 \\
\hline TMAO vs. ankle brachial index (ABI) (top quartile) & 0.0172 & 0.474 \\
\hline TMAO reductase vs. ankle brachial index (ABI) (top quartile) & 0.1347 & 0.051 \\
\hline IL-6 vs. ankle brachial index (ABI) (top quartile) & 0.382 & 0.0501 \\
\hline TMAO vs. cardio-ankle vascular index (CAVI) (top quartile) & 0.0292 & 0.349 \\
\hline TMAO reductase vs. Cardio-ankle vascular index (CAVI) (top quartile) & 0.0028 & 0.774 \\
\hline IL-6 vs. cardio-ankle vascular index (CAVI) (top quartile) & 0.0365 & 0.295 \\
\hline
\end{tabular}

